\title{
Optimization of OH Coil Spiral Topology
}

\author{
R. J. Witt \\ University of Wisconsin, Madison, WI USA
}

\section{INTRODUCTION}

Solenoids are often modelled as axisymmetric structures. In some instances, this is an inadequate representation of the solenoid. The Compact Ignition Tokamak (CIT) ohmic heating solenoid, for instance, is constructed from stacks of thick-turn pancakes. A typical pancake topology is shown in Figure 1. Each pancake consists of circular turns joined over a transition region by a transition topology to be determined. A stack is formed by placing pancakes on top of each other in such a way that current flows in the same circumferential direction in each pancake, with the transition between pancakes occurring at the inner and outer joints. Such an arrangement is also shown in Figure 1. A discussion of the non-axisymmetric behavior of the stack may be found in an accompanying paper (Witt, 1989).

The importance of the angle subtended by the transition region and its associated topology lies in two constraints imposed on the central solenoid. First, the solenoid is designed so that the last circular turn is flush against a limiting outer radius, and the outermost turn ends at the beginning of the transition region. Secondly, the solenoid provides a flux swing to drive the plasma current. To achieve a given peak field during the pulse, the number of ampere-turns must be fixed. If a very large transition angle is used, $\left(\simeq 180^{\circ}\right)$, a very smooth transition can be made, but half a turn is lost from each pancake in the solenoid; the current in the coil must be relatively high to meet the ampere-turn requirement. If the transition angle is small, only a fraction of a half-turn may be lost; the ampere-turn requirement may be met with a lower current, but a sharper transition region is required. The resistive nature of the coils makes it desirable to choose an option with lower current, but it is difficult to make the transition at small angles without introducing local regions of small radius of curvature, which results in high hoop stresses. The choice of transition angle and its associated topology is therefore driven by the best compromise between current and stress increases above axisymmetric levels.

\section{ANALYTICAL OPTIMIZATION OF TOPOLOGY}

First consider the problem of choosing the best topology for a given transition angle. The problem is illustrated in Figure 2, where the transition angle is $2 \alpha$. The goal is to find the topology, joining two circular turns of radii $a$ and $b$ and tangent to these circular turns, which gives the lowest hoop stresses of any admissible topology.

One way of approaching the problem is in terms of the deformation of circular beams. The transition or joggle region may be thought of as a curved, nearly circular beam loaded from 

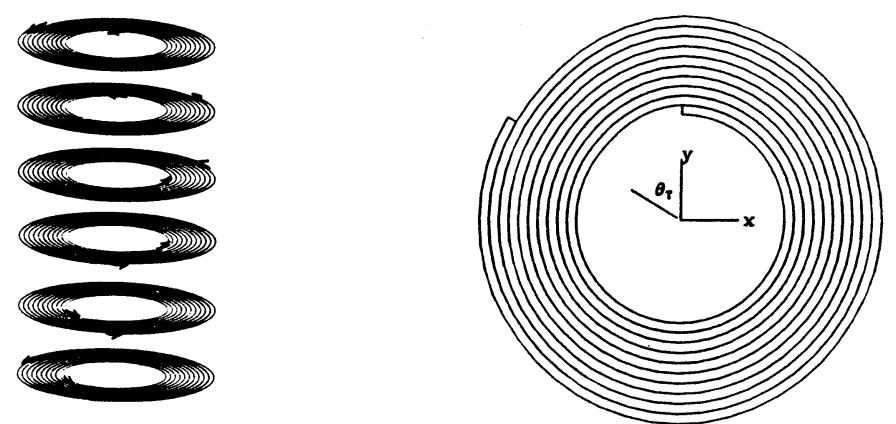

Figure 1 - Stack of Asymmetric Pancakes (Left) and Asymmetric Pancake Topology (Right)

a combination of discrete and distributed forces. Airy's stress functions may be used to describe the behavior of circular beams loaded in this fashion, with $\sigma_{\theta \theta}=\frac{\partial^{2} \varphi}{\partial r^{2}}$. For thick circular beams, the peak hoop stress occurs at the inner radius and scales as $\frac{1}{r^{n}}$, where $n$ depends on the relative contribution from the discrete and distributed loads. The actual load distributions in the transition region are difficult to determine without resorting to sophisticated finite element analyses, so the relative contribution of each component is not known a priori. Since it is expected, however, that the "best" transition will be as "circular" as possible, the "best" transition may be defined as the one which minimizes $\frac{1}{\rho^{n}}$ along its length, where $\rho$ is the local radius of curvature. It is shown below that for small departures from a circular beam the optimum transition region is independent of $n$.

The problem involves finding a function $y(x)$ passing through points $(-a \sin \alpha, a \cos \alpha)$ and $(b \sin \alpha, b \cos \alpha)$ with derivatives $\tan \alpha$ and $-\tan \alpha$ respectively (see Figure 2), such that the functional

$$
I=\int_{-a \sin \alpha}^{+b \sin \alpha}\left[\frac{y^{\prime \prime}}{\left(1+y^{\prime 2}\right)^{3 / 2}}\right]^{n}
$$

is minimized. Given a functional whose integrand takes the form $G=G\left(x, y, y^{\prime}, y^{\prime \prime}\right)$, extremals must satisfy the relation

$$
\frac{d^{2}}{d x^{2}}\left(\frac{\partial G}{\partial y^{\prime \prime}}\right)-\frac{d}{d x}\left(\frac{\partial G}{\partial y^{\prime}}\right)+\frac{\partial G}{\partial y}=0
$$

Since $F=\frac{1}{\rho^{n}}$ is not an explicit function of $x$ or $y$, this may be integrated twice to yield

$$
z^{\prime}\left(\frac{\partial F}{\partial z^{\prime}}\right)-F-C_{1} z=C_{2}
$$

where $z=y^{\prime}$. Substituting in the expression for $F$ gives a first order differential equation in $z$ :

$$
\frac{(n-1) z^{\prime^{n}}}{\left(1+z^{2}\right)^{3 n / 2}}-C_{1} z=C_{2}
$$

It is instructive to rewrite this equation as:

$$
\frac{1}{\rho}=\left(\frac{C_{1} y^{\prime}+C_{2}}{n-1}\right)^{1 / n}
$$



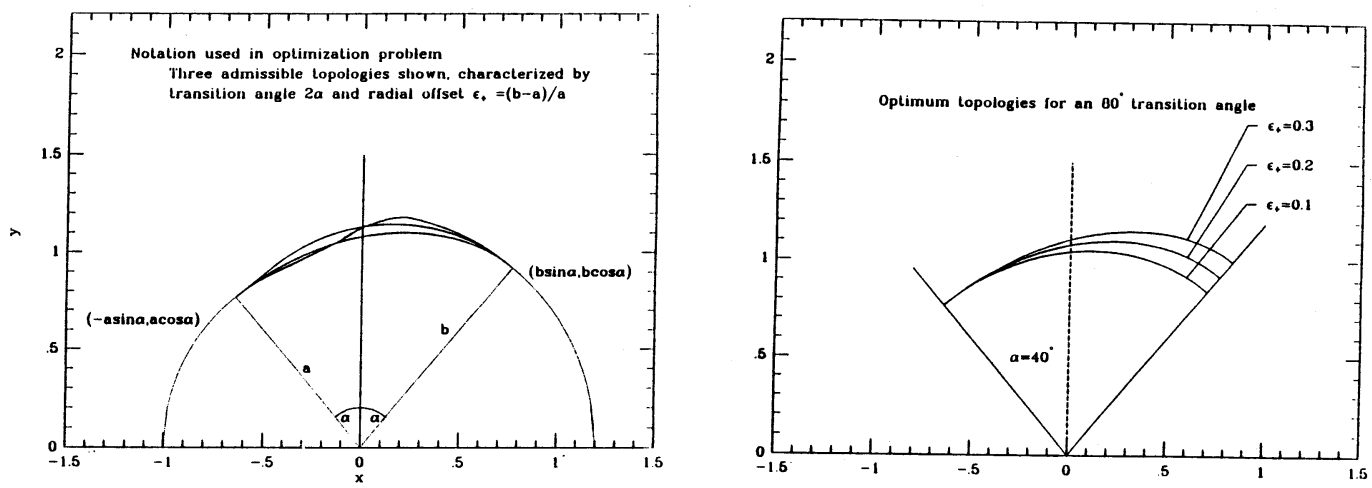

Figure 2 - Problem Definition (Left) and Optimum Topologies for an $80^{\circ}$ Transition Angle (Right)

which indicates the radius of curvature has a constant component $\left(C_{2}\right)$ and a component that depends on local position $\left(C_{1} y^{\prime}\right)$. Since it is expected that the extremal will be as "circular" as possible, a weak dependence of $\rho$ on local position is expected; this implies $\frac{C_{1} y^{\prime}}{C_{2}} \ll 1$, which allows the above equation to be linearized and integrated to give:

$$
\frac{z+\frac{1}{n} \frac{C_{1}}{C_{2}}}{\sqrt{1+z^{2}}}=\left(\frac{C_{2}}{n-1}\right)^{1 / n}\left(x+C_{3}\right)
$$

Two of the constants can be written in terms of the third by imposing the boundary conditions $z=\tan \alpha$ at $x=-a \sin \alpha$ and $z=-\tan \alpha$ at $x=b \sin \alpha$ :

$$
\begin{gathered}
\cos \alpha\left[\tan \alpha+\frac{1}{n}\left(\frac{C_{1}}{C_{2}}\right)\right]=\left(\frac{C_{2}}{n-1}\right)^{1 / n}\left(C_{3}-a \sin \alpha\right) \\
\cos \alpha\left[-\tan \alpha+\frac{1}{n}\left(\frac{C_{1}}{C_{2}}\right)\right]=\left(\frac{C_{2}}{n-1}\right)^{1 / n}\left(C_{3}+b \sin \alpha\right)
\end{gathered}
$$

Subtracting the second of the above equations from the first and rearranging,

$$
C_{2}=-(n-1) \frac{2^{n}}{(a+b)^{n}}
$$

Returning to Eqn. 5, it can be seen that the constant component of the radius of curvature is just the average of the two radii. Adding the two equations together, a new quantity $s$ is defined as:

$$
s=-\frac{1}{n} \frac{C_{1}}{C_{2}}=\frac{2 C_{3} \sec \alpha+\epsilon \tan \alpha}{(a+b)}
$$

where $\epsilon=b-a$ so that Eqn. 6 now becomes

$$
\frac{z-s}{\sqrt{1+z^{2}}}=-2 \frac{x+C_{3}}{a+b}
$$

Note that $s$ and the resulting differential equation are independent of $n$; the equation can be rearranged to give:

$$
\frac{d y_{+}}{d x_{+}}=\frac{s \pm 2 x_{+} \sqrt{s^{2}+1-4 x_{+}^{2}}}{1-4 x_{+}^{2}}
$$



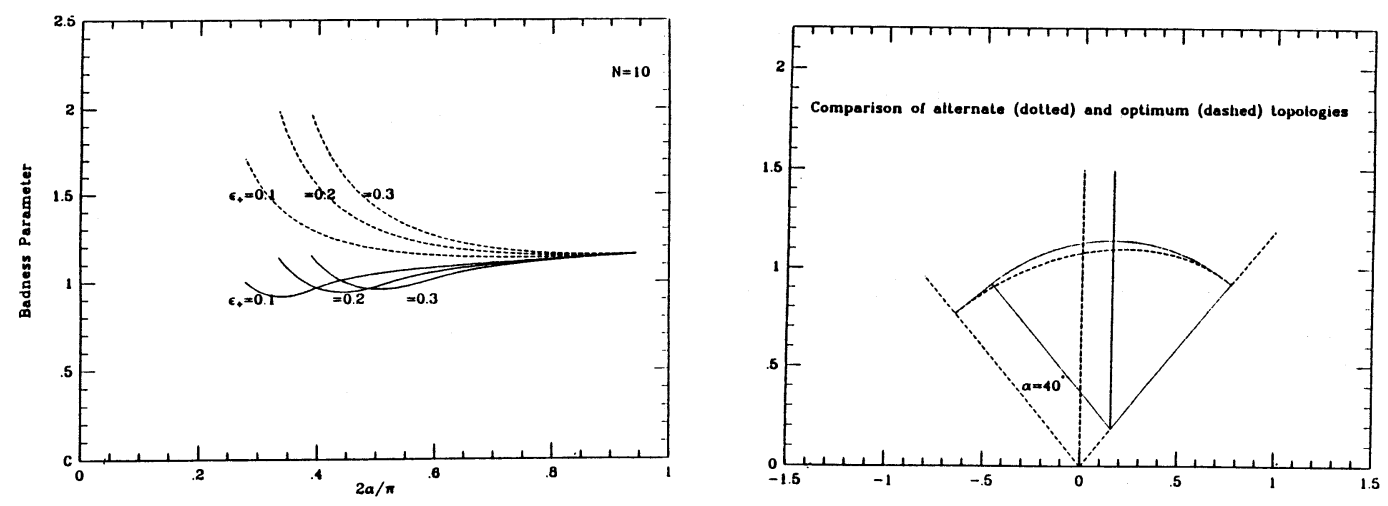

Figure 3 - Badness Parameter vs. Transition Angle (Left) and Comparison of Optimum and Alternate Topologies (Right)

where $y_{+}=y /(a+b)$ and $x_{+}=\left(x+C_{3}\right) /(a+b)$. The negative root of this equation is the relevant one for this application.

Results for $\alpha=40^{\circ}$ and several $\epsilon_{+}(=\epsilon / a)$ are also shown in Figure 2. Very smooth curves can be found at large $\alpha$ for all $\epsilon_{+}$, but not for small $\alpha$ and large $\epsilon_{+}$. Mathematically, it is clear that when $\alpha \rightarrow 0$ as $\epsilon_{+}$remains finite, it is impossible to connect the two points with the appropriate boundary conditions using the same family of curves characteristic of large $\alpha$ and small $\epsilon_{+}$. The curves can't be joined appropriately at small $\alpha$ without introducing an inflection point, and this is represented by the need to switch from the negative root of Eqn. 12 to the positive root. A complete set of solutions over all $\left(\alpha, \epsilon_{+}\right)$can therefore not be found with this approach.

\section{ANALYTICAL COMPARISON OF TRANSITION ANGLES}

Within the range of $\left(\alpha, \epsilon_{+}\right)$that the method is valid, a best transition angle may be found by minimizing the penalties associated with both factors. A "badness parameter" is defined as:

$$
B\left(\epsilon_{+}, \alpha\right)=\left[\frac{\int_{-\sin \alpha}^{\left(1+\epsilon_{+}\right) \sin \alpha}\left(\frac{\rho_{o}}{\rho}\right)^{n} d\left(\frac{x}{a}\right)}{\left(2+\epsilon_{+}\right) \sin \alpha}\right]\left(\frac{N}{N-\frac{\alpha}{\pi}}\right)^{m}
$$

where $\rho_{o}=(a+b) / 2$. The best angle is then the one with the smallest "badness". The term in [] is the functional $I$ defined in Eqn. 1 normalized to the path length, and is defined as the stress factor. For any piece of a circular arc of radius $\rho_{o}$, the term in [] is one. The term in () is a penalty factor which reflects the fact that the current through the turns must be increased as more of the outside turn is lost to keep the number of ampere-turns constant. $N$ in () is the nominal number of turns and is usually 8,9 or 10. The exponents $n$ and $m$ are not clearly defined; for purposes of making comparisons $n=m=3$.

The badness parameter is useful in that it illustrates some general features of the behavior as a function of transition angle. Values of the badness parameter are plotted vs $\alpha$ in Figure 3 for $m=3$ and $n=3$ for $N=10$. Minima are clearly visible, with smaller optimum angles at smaller $\epsilon_{+}$. As the transition angle decreases from $180^{\circ}$ through $90^{\circ}$, the transition can be made without introducing local regions of small radii of curvature. The badness parameter decreases as the reduction in the current penalty factor dominates the behavior. As the transition angle becomes smaller, however, it becomes increasingly difficult to make the transition without these local regions of small radii of curvature, and the stress factor begins to dominate. The minima occur at smaller angles as $\epsilon_{+}$goes to zero, as the transition can be made at smaller angles without introducing sharp transitions in the topology. 

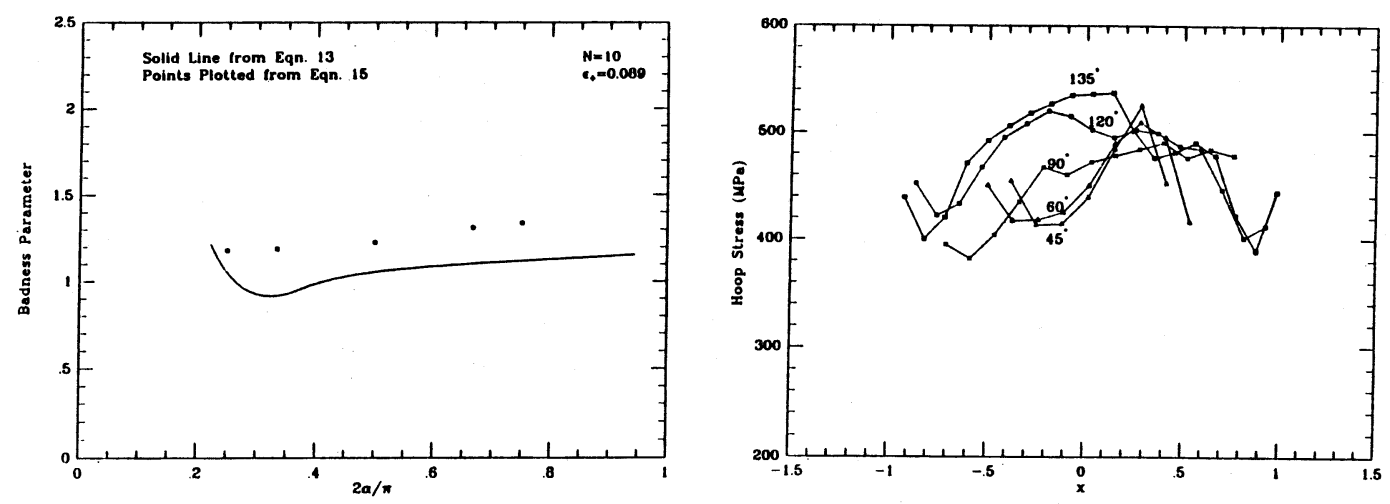

Figure 4 - Analytical and Computed Badness (Left) and Hoop Stress Distributions in Joggle (Right)

The sensitivity of the badness parameter to deviations from the optimum topology has also been investigated. A second set of badness parameters is plotted in Figure 3 for a particular type of topology consisting of a straight section (infinite radius of curvature), followed by a pivot of constant radius over the remaining portion of the transition. This toplogy is also shown in Figure 3 with the optimum topology for $\alpha=40^{\circ}$. The new topology is represented by $y_{n}(x)=y_{+}(x)+\delta y(x)$. Within the range of $\alpha$ where the $\delta y$ are of the same order, the difference in the badness parameters becomes a measure of the sensitivity of the results to deviations from the optimum topology.

It can be shown that for the straight/pivot topology the stress factor in the badness parameter is:

$$
B^{*}=\left(2+\epsilon_{+}\right)^{2} \frac{\sin ^{4} \alpha}{\left[1-\left(1+\epsilon_{+}\right)\left(\cos ^{2} \alpha-\sin ^{2} \alpha\right)\right]^{2}}
$$

At large transition angles, values of $B^{*}$ are very close to those associated with the optimum topology. As the transition angle becomes smaller, however, differences between $B^{*}$ and the optimum $B$ become more pronounced. The badness parameter becomes more sensitive to deviations from the optimum topology as the transition angle decreases, suggesting that the stress field becomes strongly dependent on the topology.

\section{COMPARISON OF ANALYTICAL AND COMPUTATIONAL RESULTS}

Because of the uncertainty in the badness parameter, a proper evaluation of the best transition angle must be made on the basis of a computational model. For the specific case of the 2.1 meter CIT PF2 $\left(N=10 ; \epsilon_{+}=0.089\right)$, the badness parameter is plotted vs. transition angle in Figure 4. The points plotted are calculated from finite element analyses by using an alternate definition of the stress factor in the badness parameter:

$$
B_{F E}=\left[\frac{\int_{-\sin \alpha}^{\left(1+\epsilon_{+}\right) \sin \alpha} \frac{\sigma_{h}\left(\frac{x}{a}\right)}{\sigma_{o}} d\left(\frac{x}{a}\right)}{\left(2+\epsilon_{+}\right) \sin \alpha}\right]\left(\frac{N}{N-\frac{\alpha}{\pi}}\right)^{m}
$$

where $\sigma_{h}$ is the local hoop stress along the innermost fiber of the inner turn in the transition region, and $\sigma_{o}$ is the characteristic hoop stress of a purely axisymmetric structure (400 $\mathrm{MPa}$ in this case). The distributions of $\sigma_{h}$ are also sketched in Figure 4 for various transition angles.

Differences between the analytical and computed badness can be explained in terms of these stress distributions. When the transition angle is large, the transition region does not look like 
a thick circular beam anywhere along its length. Airy's stress functions contain a number of terms, and for thin beams each of the terms is of the same order of magnitude. The badness parameter, on the other hand, is defined on the basis of a single term dominating the behavior. These differences lead to the differences in magnitude between the analytical and computed values in Figure 4. If the analytical badness parameter were representative of the true behavior at large transition angles, there would exist regions, where $\rho>\frac{a+b}{2}$, in which the hoop stress falls below the axisymmetric level of $400 \mathrm{MPa}$. The plots in Figure 4 illustrate that, while there are regions where the stress falls below $400 \mathrm{MPa}$, most regions are well above the axisymmetric value. Computed values of badness (Eqn. 15) are therefore larger than the analytical values (Eqn. 13).

Even though finite element analyses may be used to determine the badness parameter as a function of transition angle, additional factors may be involved in the choice of best angle. Fatigue failure is caused by cracks growing under applied load, which is a local phenomenon. The best transition angle may be the one with the lowest peak stress in the joggle region. Of the angles considered, Figure 4 indicates that a $90^{\circ}$ angle would be best under this criterion.

It is then natural to ask whether a better topology might be found by changing the optimization procedure to minimize local maxima. Given that the problem is by definition a global one (literally how to connect point A to point B), it is not clear how to formulate such a procedure. The global optimization procedure based on Eqn. 1 does, however, tend to exclude regions with small radii of curvature because of the penalty associated with such regions. The result from Eqn. 5, that the radii of curvature are equally distributed on either side of the average radius of curvature, reinforces the notion that the topology presented here is also optimum in the local sense.

\section{CONCLUSIONS}

A method has been proposed for designing the transition region for thick-turn pancakes in solenoids. The method is based on calculus of variations and yields a first order differential equation describing the transition region topology. This optimum topology has an average radius of curvature equal to the average of the two circular turn radii; the radius of curvature at the inner turn is found to be greater than this average, and the radius of curvature at the outer turn is found to be less than this average by the same amount. The method breaks down when the difference in circular turn radii becomes very large at small transition angles.

Within the limits that the method is valid, a "badness parameter" can be used to choose a best transition angle. This parameter is best used to illustrate general qualitative behavior, as exponents embedded in the parameter are not clearly defined, and factors other than the global behavior of the joggle may influence the choice of transition angle. The parameter indicates that the optimum angle becomes smaller as the difference between the two radii becomes smaller, and that the design becomes more unforgiving to deviations from the optimum topology as the transition angle decreases. Comparison of the analytical badness parameter with a similar parameter calculated from finite element analyses indicates that the badness parameter is indeed representative of the qualitative changes in joggle stress fields with transition angle.

\section{REFERENCES}

Witt, R.J. and Bobrov, E.S. (1989). Non-Axisymmetric Response of OH Coils to Lorentz Forces. SMiRT 10. 